\title{
Critical Illness Polyneuropathy and Myopathy in COVID-19 Patients: A Prospective Observational Intensive Care Unit Cross-Sectional Cohort Study
}

Robert Frithiof

Uppsala University: Uppsala Universitet

Elham Rostami

Uppsala Universitet

Eva Kumlien

Uppsala University: Uppsala Universitet

Johan Virhammar

Uppsala University: Uppsala Universitet

David Fällmar

Uppsala University: Uppsala Universitet

Michael Hultström

Uppsala University: Uppsala Universitet

Miklós Lipcsey

Uppsala University: Uppsala Universitet

Nicholas Ashton

University of Gothenburg: Goteborgs Universitet

Kaj Blennow

University of Gothenburg: Goteborgs Universitet

Henrik Zetterberg

Goteborg University: Goteborgs Universitet

Anna Rostedt Punga ( $\square$ anna.rostedt.punga@neuro.uu.se )

Uppsala Universitet https://orcid.org/0000-0002-2178-9413

\section{Research}

Keywords: critical illness, neuropathy, myopathy, CINM, Covid-19, SARS-CoV-2, NFL

Posted Date: September 22nd, 2020

DOI: https://doi.org/10.21203/rs.3.rs-78038/v1 
License: (c) (i) This work is licensed under a Creative Commons Attribution 4.0 International License. Read Full License 


\section{Critical illness polyneuropathy and myopathy in COVID-19 patients: a prospective observational intensive care unit cross-sectional cohort study}

Robert Frithiofı\#, PhD; Elham Rostami2, s\#, PhD; Eva Kumlien3, PhD; Johan Virhammar3, PhD; David Fällmar6, PhD; Michael Hultström1, 7, PhD; Miklós Lipcseyıb, PhD; Nicholas Ashton8, 9, 10, 11, PhD; Kaj Blennow8, 12, PhD; Henrik Zetterberg8, 12, 13, 14, PhD; Anna Rostedt Punga4*, PhD

1 Department of surgical sciences, Anesthesiology and Intensive Care Medicine, (bHedenstierna Laboratory), Uppsala University, Uppsala, Sweden Department of neuroscience, 2 Neurosurgery, 3 Neurology and 4 Clinical Neurophysiology, Uppsala University, Uppsala, Sweden ${ }_{5}$ Department of neuroscience, Karolinska Institutet, Stockholm, Sweden ${ }_{6}$ Department of surgical sciences, Radiology, Uppsala University, Uppsala, Sweden 7Department of Medical Cell Biology, Integrative Physiology, Uppsala University, Uppsala, Sweden.

8Department of Psychiatry and Neurochemistry, Institute of Neuroscience and Physiology, the Sahlgrenska Academy at the University of Gothenburg, Mölndal, Sweden 9Wallenberg Centre for Molecular and Translational Medicine, University of Gothenburg, Gothenburg, Sweden 10King's College London, Institute of Psychiatry, Psychology \& Neuroscience, Maurice Wohl Clinical Neuroscience Institute, London, UK 11NIHR Biomedical Research Centre for Mental Health \& Biomedical Research Unit for Dementia at South London \& Maudsley NHS Foundation, London, UK ${ }_{12}$ Clinical Neurochemistry Laboratory, Sahlgrenska University Hospital, Mölndal, Sweden 13Department of Neurodegenerative Disease, UCL Institute of Neurology, London, UK 
14UK Dementia Research Institute at UCL, London, United Kingdom

\# shared first authorship;

*Corresponding author:

Professor Anna Rostedt Punga

Address: Department of Neuroscience, Clinical Neurophysiology, Uppsala University, Uppsala University Hospital; entrance 85, 3rd floor, 75185 Uppsala, SWEDEN

Telephone: +46-18-4714941

Fax: + 46-18-504768

email: anna.rostedt.punga@neuro.uu.se

Word count: 3338 words

Keywods: critical illness, neuropathy, myopathy, CINM, Covid-19, SARS-CoV-2, NFL

\begin{abstract}
Background: Several reports on neurological complications associated with SARS-CoV-2 infection have been published. However, systematic description on intensive care unit acquired weakness (ICUAW) are still missing.
\end{abstract}

Methods: The objective was to determine the incidence and characteristics of critical illness polyneuropathy (CIN) and myopathy (CIM) in patients with severe COVID-19. We also aimed to describe the electrophysiological features and their relation to plasma biomarkers for neuronal injury. This was a prospective observational intensive care unit cohort study. All adult patients admitted to the general intensive care unit (ICU) at Uppsala University Hospital, Uppsala, Sweden, between March 13 and June 8, 2020 were screened for inclusion. 
Patients with PCR confirmed COVID-19 were included. All patients were admitted to intensive care treatment due to severe COVID-19, including intravenous anaesthesia, opioid anaelgesia, neuromuscular blockade and mechanical ventilation. Associations of clinical, electrophysiological (sensory and motor conduction studies and electromyography) and biomarker data [neurofilament light chain (NfL), glial fibrillary acidic protein (GFAp) and tau] were studied between COVID-19 patients who developed CIN/CIM and those who did not.

Results: 111 COVID-19 patients were included, 11 (11 males, mean age: 64 years) developed CIN/CIM whereas 100 (74 males, mean age: 61 years) did not (non-CIN/CIM). The CIN/CIM incidence was higher in COVID-19 patients compared to a general ICUpopulation treated during $2019(9.9 \%$ vs 3.4\%). In particular CIN was more frequent in the COVID-19 ICU cohort (50\%) compared with the non-COVID-19 ICU cohort $(0 \%, \mathrm{p}=0.008)$. NfL and GFAp levels were higher in the CIN/CIM group both at the early ( $<9$ days) and late time points $(>11$ days) compared with the non-CIN/CIM group (both $\mathrm{p}=0.001)$ and correlated with nerve amplitudes.

Conclusions: CIN/CIM, in particular CIN, were more prevalent among COVID-19 patients than an ICU treated control cohort and should be considered in the differential diagnostic workup and the further rehabilitation of COVID-19 patients. COVID-19 patients who later developed ICUAW had significantly higher NfL and GFAp in the early phase of ICU care, which suggests their potential as predictive biomarkers.

Trial registration: The protocol of the study was registered (ClinicalTrials ID: NCT04316884). Mechanisms for Organ Dysfunction in Covid-19 (UMODCOVID19). Registered on March 18, 2020. 


\section{BACKGROUND}

Neurological manifestations of severe acute respiratory syndrome coronavirus 2 (SARS$\mathrm{CoV}-2$ ) are increasingly recognized as a major complication with potential long-term consequences for both patients and the health care system[1]. The first study from Wuhan reported neurological symptoms in approximately $36 \%$ of confirmed coronavirus disease 2019 (COVID-19) cases. However, the clinical investigation were mainly based on subjective symptoms and clinical/laboratory analyses[2]. Patients with severe COVID-19 infections are at risk to develop neurological manifestations, especially acute cerebrovascular disease, disturbed consciousness and skeletal muscle injury[2]. Manifestations in the peripheral nervous system have been less frequently reported but require specific investigations that may not always have been available or practical in the setting of the ongoing pandemic. In the previous severe acute respiratory syndrome (SARS) infection pandemic in 2004, a case series of four patients highlighted possibly coexistent critical-illness polyneuropathy and/or myopathy, suggesting further studies to determine a potential relationship between SARS coronavirus and neuromuscular problems [3]. Acute inflammatory polyneuropathy, e.g., Guillain-Barré syndrome (GBS), has been described in 19 COVID-19 patients worldwide[46], but to our knowledge intensive care unit acquired weakness (ICUAW) has not yet been characterized in COVID-19 patients.

Important pathophysiological mechanisms of ICUAW are critical illness neuropathy (CIN) and myopathy (CIM), where patients present with flaccid limb muscle weakness and failure to wean from the ventilator [7]. One of the medical challenges with COVID-19 patients is the high number of patients requiring prolonged mechanical ventilation in the intensive care unit (ICU) in combination with unusually high sedation requirements that both predispose to ICUAW [8]. CIN and CIM are important to identify, since survivors often present with 
severe residual disability and persistent exercise limitations several years afterwards [9-11]. There is further a clear distinction between outcome in CIN versus CIM, where patients with CIN have a slower or incomplete recovery, and higher mortality rate, whereas patients with CIM often show complete recovery within 6 months [12]. Thus, it would be highly valuable to predict COVID-19 patients at risk of developing CIN/CIM and secondly distinguish between the two if possible. So far, only one case report of a patient with CIM has been published [13] and no reports have to our knowledge described any cases of CIN associated with COVID-19. Except for electrophysiological parameters that classify CIN or CIM, increased plasma concentration of the axonal injury marker neurofilament light (NfL) has been described elevated in patients with ICUAW [14].

The aim of this study was to conduct a prospective study of all COVID-19 patients admitted to the ICU to characterize the ICUAW into CIN and CIM, as well as to determine potential correlating plasma biomarkers for neuronal injury. In order to understand the potential influence of COVID-19 on incidence of CIN/CIM, we also compared the number of cases with retrospective electrophysiological data for ICU patients during 2019.

\section{METHODS}

\section{Study design and patients}

The prospective observational part of the study was performed at the general ICU at Uppsala University Hospital, at the time only treating suspected or confirmed COVID-19 patients. All adult patients admitted to the ICU between March 13, and June 8, 2020 were screened for inclusion (supplementary figure 1). Clinical data and medical history of the cohorts were collected from the electronic medical record and presented in agreement with the STROBE criteria. The protocol of the study was registered (ClinicalTrials ID: NCT04316884). The COVID-19 cohort (COVID-19) was defined as hospitalized patients with PCR confirmed 
positive SARS-CoV-2 [15]. ICU controls (non-COVID-19 patients) were treated for other disorders during the same time period and referred for electrophysiological evaluation due to ICUAW [referring diagnosis to the department of clinical neurophysiology of either CIN (ICD10 code G63.9) or CIM (ICD-10 code G72.9)].

In order to compare the incidence rate of ICUAW and CIN/CIM during 2020 with a year prior to COVID-19, all cases admitted to the ICU were noted, in addition to diagnoses of those that were referred to electrophysiological evaluation.

The study was approved by the National Ethical Review Authority (EPM; No. 2020-01623). Informed consent was obtained from the patient, or next of kin if the patient was unable give consent. The Declaration of Helsinki and its subsequent revisions were followed. The protocol of the study was registered (ClinicalTrials ID: NCT04316884). Electrophysiological data collected from COVID-19 patients and ICU controls were approved by the Ethical Review Board (case no 2015/105 and 2020-03377).

\section{Electrophysiological examinations and parameters}

Motor NCS (NCS) were performed in the median and ulnar nerve of one arm and in the fibular and tibial nerves of both legs; parameters including distal motor latency, conduction velocity, amplitude and amplitude decay between proximal and distal stimulation (conduction block assessment), as well as F responses. Orthodromic sensory NCS were done for the ulnar and radial nerves of one arm as well as the sural and superficial fibular nerves in both legs with parameters including distal latency, amplitude and conduction velocity. Combined motor and sensory amplitude scores were calculated as the mean amplitude of all recorded motor nerves divided by amount of nerves examined. 
Low frequency $(3 \mathrm{~Hz})$ repetitive nerve stimulation was done of the ulnar nerve with recording over the adbuctor digiti quinti muscle, to eliminate decremental response as a lingering neuromuscular blocking effect of medications.

Electromyography with a concentric needle electrode was performed in limb and facial skeletal muscles in order to detect abnormal spontaneous activity at rest (fibrillations and/or positive sharp waves) and if possible, if the patients could collaborate, analyse the presence of myopathic (polyphasic, short duration, low amplitude) motor unit potentials.

\section{Diagnostic criteria of CIN and CIM}

Diagnostic criteria for both CIN and CIM included:

i. the patient was critically ill (multiorgan dysfunction and failures) and

ii. limb weakness or difficulty weaning from ventilator after non-neuromuscular causes such as heart and lung disease have been excluded.

For CIN, additional diagnostic criteria included: iii. electrophysiological evidence of axonal motor and sensory polyneuropathy, iv. absence of a decremental response on repetitive nerve stimulation (RNS)[16].

For CIM, additional criteria included: iii. CMAP amplitudes $<80 \%$ of the lower limit of normal in $\geq 2$ nerves without conduction block; iv. sensory nerve action potential amplitudes $>80 \%$ of the lower normal limit; v. Needle electromyography with short duration, low-amplitude motor unit potentials with early or normal full recruitment, with or without fibrillation potentials in conscious and collaborative patients; or increased CMAP duration or reduced muscle membrane excitability on direct muscle stimulation in noncollaborative patients; vi. absence of a decremental response on RNS[17]. 


\section{Analysis of plasma biomarkers for neuronal injury and astrocytic activation}

Plasma samples were collected at different time points in the ICU ward, early $(<9$ days from admission) and at a later stage ( $>11$ days from admission). The results from COVID-19 patients with CIN/CIM ( $\mathrm{N}=11)$ were compared with those from intubated COVID-19 patients treated $>12$ days in the ICU who did not develop CIN/CIM (N=7). Levels of neurofilament light chain (NfL), tau and glial fibrillary acidic protein (GFAp) were measured using Single molecule array (Simoa) technology (Quanterix, Billerica, MA), as previously described[18] using a single batch of reagents. Intra-assay coefficients for internal control samples were below $7 \%$ for all analytes.

\section{Statistical analysis}

Data are represented as median (IQR) or n (\%). Mann-Whitney U test was used for continuous parameters and the chi-square test for categorical parameters.

The plasma biomarker data were log transformed to achieve near normal distribution and analyzed with two-way repeated measures analysis of variance (ANOVA; CIN/CIM y/n x time) followed by Fishers LSD test or Bonferroni-corrected planned comparisons when suitable. Correlation analysis between plasma biomarkers (tau, GFAp, NfL) with NCS parameters were done with Spearman Rank correlation. Statistical comparison of descriptive variables (diagnoses) between the groups was done with Chiz-test. A p-value of $<0.05$ was considered significant. 


\section{RESULTS}

\section{Characteristics of the ICU patients}

After screening of 122 patients, a total of 111 COVID-19 patients were included in the study. $23 \%$ of patients died within the first 2 weeks and of the remaining patients, $11(9.8 \%)$ developed CIN/CIM and 100 did not ("non-CIN/CIM") (supplementary figure 1). Demographic characteristics and comorbidities of these patients are displayed in table 1. There was a significant overrepresentation of men in the CIN/CIM patient cohort as well as a higher BMI (table 1). There was no significant difference in age, blood group or preexisting comorbidities between the groups (table $1 ; \mathrm{p}>0.05$ ). On admission, there were no significant differences in vital parameters or number of days with the COVID-19 infection (table 2; $p>$ 0.05). The CIN/CIM cohort had a longer duration of stay in the ICU (median: 27 versus 7 days; $p<0.001)$, more thromboembolic events $(p=0.006)$, more days with invasive ventilation (median: 18 versus $8 ; \mathrm{p}<0.001$ ), more frequent vasoactive medication (median: 14 versus $5 ; p<0.001$ ) and renal replacement therapy (median: 21 versus $6 ; p=0.006$; table 2). Furthermore, patients who developed CIN/CIM were more frequently treated with anesthetics, opioid analgesics and neuromuscular blocking agents (table 2; $p<0.001$ ). No difference was seen in ICU mortality or 30-day mortality between the groups (table 2; p > $0.05)$.

\section{Characteristics of the ICU weakness in COVID-19 compared to non-COVID-19} patients

During the selected time period of 6 months, 14 COVID-19 patients and 10 non-COVID (control) patients from the ICU at Uppsala University Hospital had electrophysiological examination due to ICUAW. All of the patients referred for electrophysiological evaluation 
due to suspicion of ICUAW had reduced muscle tone and none had any signs of decrement at low frequency RNS, thus eliminating neuromuscular blockade as a cause of the muscle weakness. The underlying diagnoses in the ICU control cohort were subarachnoidal hemorrhage $(\mathrm{N}=4)$, sepsis $(\mathrm{N}=2)$, pneumonia $(\mathrm{N}=1)$, herpes encephalitis $(\mathrm{N}=1)$, meningitis $(\mathrm{N}=1)$ and subdural hematoma $(\mathrm{N}=1)$. There were significantly more men $(94 \%)$ in the COVID-19 cohort compared to the ICU-ctrl group (30\%; $<<0.001$; table 3$)$. The age was comparable between the groups $(\mathrm{p}=0.42)$. A neurophysiological pattern of CIN/CIM was seen in $11(79 \%)$ of the COVID-19 cohort and in seven ICU control patients (70\%, all CIM; table 1). A clear CIN pattern with axonal sensorimotor polyneuropathy was seen in as many as seven $(50 \%)$ patients in the COVID-19 cohort, whereas this was not seen in any patients in the ICU control cohort $(\mathrm{p}=0.008)$. Instead, a distinct CIM pattern was slightly more commonly encountered among the ICU control patients (table 3; $\mathrm{p}=0.05$ ). Spontaneous activity on EMG was seen in nine of the 14 examined COVID-19 cases. F responses were absent in the tibial nerve of 8 (57\%) COVID-19 patients compared with three (30\%) ICU control patients.

All patients but one in the COVID-19 cohort (13/14) were examined with a CT scan of the head at least once, and seven of these were also examined with an MRI of the brain. Four patients had pathological neuroradiological findings that did not explain the peripheral weakness: acute necrotizing encephalopathy $(\mathrm{N}=1)$, bilateral infarcts in deep white matter $(\mathrm{N}=1)$, unspecific white matter changes with microhemorrhage $(\mathrm{N}=1)$, and numerous bilateral microhemorrhages in white matter $(\mathrm{N}=1)$. The remaining patients had normal neuroradiological findings.

\section{Comparison of incidence data on ICUAW 2019}


Next, we aimed at comparing the incidence rate of ICUAW with a year prior to COVID-19. During 2019, 865 general ICU-patients were treated in the same ward. Out of these patients, 29 (15 male; mean age: 63 years) were referred for NCS and EMG based on the question of CIN/CIM due to ICUAW and 22 (2.5\%) were diagnosed with CIN and/or CIM. Eighteen patients $(82 \%)$ had CIM and four (18\%) had CIN.

\section{Analysis of blood biomarkers for neuronal injury and astrocytic activation}

Analysis of biomarkers for neuronal injury and astrocytic activation was done from early plasma samples, within 9 days (median: 4, range: 3-9), and late, after 11 days (median: 16, range: 11-42 days) from admission to the ICU. All patients analyzed had severe COVID-19, required invasive ventilation and were treated in the ICU for $>12$ days. The seven patients who did not develop CIN/CIM had fewer days with invasive ventilation and renal replacement therapy compared with the CIN/CIM group. Besides that, there were no significant differences with regards to demography and clinical parameters (supplementary tables 1 and 2). NfL levels were significantly higher in the CIN/CIM group both at the early and late time points $(\mathrm{p}<0.001$ and $\mathrm{p}=0.03$, respectively; Figure $1 \mathrm{~A})$. NfL levels were also assessed against ICU time (Supplementary figure 2). Higher NfL concentration correlated

with longer ICU time for the CIN/CIM group ( $p=0.02 ; \mathrm{R}=0.4)$ and also for the non-CIN/CIM group ( $\mathrm{p}=0.005 ; \mathrm{R}=0.62$ ). GFAp levels were significantly higher in the CIN/CIM group at both early $(p=0.04)$ and late $(p=0.02)$ timepoints (Figure 1B), with no significant effect of time in the ICU (data not shown). Tau were significantly increased in the CIN/CIM cohort compared to patients with non-CIN/CIM ( $\mathrm{p}=0.04$ ) when considering all samples but not when divided in early and late timepoints ( $p=0.08$ and $p=0.06$, respectively; Figure $1 \mathrm{C}$ ). 


\section{Correlation between plasma biomarkers and NCS parameters comparing a subgroup of non-CIN/CIM with CIN/CIM}

In a cohort of 13 COVID-19 patients with plasma biomarker results from the same time point as the electrophysiological work-up, six patients were diagnosed with CIN, four patients were diagnosed with CIM and three patients had normal electrophysiological data. The combined motor amplitude (CMA) score was significantly lower in the CIN/CIM cohort (median: 2.5, range 1.3-3.5) than in the non-CIN/CIM cohort (median: 5.2, range 4.4-8.4; $p=0.0070$; Figure 2A). In addition, the combined sensory amplitude (CSA) score was lower in the CIN/CIM cohort (median: 4.5, range 2.7-6.4) than the non-CIN/CIM group (median: 12.3, range 9.814.3; $\mathrm{p}=0.007$; Figure 2B). A correlation analysis was done between levels of GFAp, NfL and tau with sensory and motor nerve parameters from the NCS data. For GFAp, there was a significant correlation with $\mathrm{CMA}(\mathrm{R}=,-0.72 ; \mathrm{p}=0.007)$, i.e., the more severe axonal motor neuropathy (lower CMA score), the higher the GFAp levels (Figure 3A). For NfL, there was a similar trend towards significant correlation with CMA $(\mathrm{R}=-0.511 ; \mathrm{p}=0.078)$ and a correlation with fibular nerve motor amplitude $(\mathrm{R}=-0.64 ; \mathrm{p}=0.022)$. For tau, there was a significant correlation with sural nerve sensory amplitude $(\mathrm{R}=-0.59 ; \mathrm{p}=0.036)$ and $\mathrm{CSA}(\mathrm{R}=-$ $0.63 ; \mathrm{p}=0.024$; Figure 3B).

\section{DISCUSSION}

There was a strikingly higher incidence of CIN in the COVID-19 ICU cohort compared with the non-COVID-19 ICU cohort during the enrolment period. This is in contrast to the overall literature, which states that CIM is more common than CIN [19]. One recent case of CIM as a likely consequence of SARS-CoV2 infection was published [13], but to our knowledge no cases of CIN have yet been reported. Speculations have also been made regarding increased 
number of CIM patients in COVID-19 cohorts since propofol is considered a risk factor for CIM [20]. Since patients with CIN has a slower recovery or do not recover in addition to higher mortality rates, this is an important ICU consequence to consider for the COVID-19 patients. Depending on the underlying comorbidities, CIN and CIM can be common in critically ill patients. In patients suffering from sepsis with multiorgan failure the incidence can be as high as $100 \%$ [21]. In patients with ARDS, the incidence is about 20-60\% [22, 23] and in general about 20\% [24]. The major risk factors associated with CIN/CIM are multiple organ failure, muscle inactivity, hyperglycemia and the use of corticosteroids, sedation and neuromuscular blocking agents [25].

The exact global incidence of CIN and CIM remains unknown due to a wide variation in different patient populations, risk factors and in the timing of assessment[26]. Compared to the prior year 2019, when COVID-19 patients were not seen in the ICU, CIN/CIM was more frequently diagnosed in the COVID-19 cohort. Another difference was the relatively high percentage of COVID-19 ICU patients with ICUAW who had CIN, with sensorimotor axonal polyneuropathy, whereas CIM was more common among the non-COVID-19 patients. Clinically, it is very difficult to distinguish CIN from CIM in patients with ICUAW; hence, detailed NCS and EMG are essential for this classification. Theoretically, sensory nerve amplitudes could be lower in cases of edema, however edema was not observed in our COVID-19 cohort. Immobility has severe effects on skeletal muscle, and is a risk factor for muscle weakness and wasting; however this is characterized by normal motor and sensory nerve conduction and EMG studies [7]. There are different plausible explanations for a higher incidence of CIN in the COVID-19 patients. The most likely explanation is the increased number of days with mechanical ventilation, requiring an accumulated higher 
amount of anesthesia and analgesia. Actually, almost all COVID-19 patients who were intubated for $>2$ weeks developed ICUAW and the majority of them developed CIN/CIM. Another is severe respiratory failure prompting the use of neuromuscular blocking agents to improve oxygenation during invasive ventilation. The found absence of $\mathrm{F}$ waves in a higher proportion in the COVID-19 cohort may result from inexcitability of spinal motor neurons [27]. Nevertheless, NCS showed clear signs of axonal sensorimotor degeneration.

Microcirculatory changes have been proposed to have a key role in causing distal axonopathy in CIN [16]. It is debated, though, whether axonal inexcitability and degeneration are different disorders or merely two processes in the same disorder, with inexcitability, due to inactivation of sodium channels, being a reversible event preceding axonal degeneration in the case of persisting hypoxia[28].

There are no available blood biomarkers to distinguish CIN or CIM from normal NCS in patients with ICUAW. A previous pilot study observed that peak neurofilament levels had good discriminative power for ICUAW but this occurred at a later stage and first after the muscle strength assessment [14]. In a recent study higher plasma levels of NfL and GFAp were correlated with severity of COVID-19[18]. All our included subjects suffered from severe COVID-19, but still the CIN/CIM patients had higher plasma NfL levels. In the current study, we observed significantly higher NfL levels in the CIN/CIM group, both at the early and late time points, prior to onset of ICUAW. NfL is a neuronal cytoplasmic protein, which is particularly highly expressed in myelinated axons. Levels of NfL in the blood proportionally increase to the degree of axonal damage in several neurological disorders, including multiple sclerosis, amyotrophic lateral sclerosis and Alzheimer's disease [29], but also in peripheral neuropathies [30-32]. Several patients in our control group had underlying cerebral insults that could affect the biomarker levels but still the CIN/CIM showed 
significantly higher levels of NfL. A larger study would be needed to confirm the potential of NfL to determine peripheral axonal loss in CIN or other polyneuropathies. GFAp is a suggested astrocyte biomarker for glial activation, with elevated levels in Alzheimer's disease [33]. Early high levels of GFAp were also found in the CIN/CIM group, which also correlated with motor amplitude score, indicating that GFAP could have potential as marker for axonal pathology in myelinated nerves. Tau levels were slightly higher among CIN/CIM patients, which is interesting since tau mainly is a biomarker in cerebrospinal fluid in neurodegenerative brain disorders [34]. We found a correlation between tau and the sensory amplitude score, which is in line with a recent study indicating that serum levels of phosphorylated tau may be a useful biomarker for severe axonal injury due to traumatic spinal cord injury [35].

\section{Strengths and limitations}

This observational study has several strengths. First, we prospectively included all patients to the ICU in an unbiased manner and recorded all vital parameters for the duration of the ICU care. All patients referred for electrophysiological evaluation were examined according to the same standardized protocol with NCS and EMG.

This observational study also has several limitations that may affect the generalizability of our results. First, referrals to NCS and EMG were strictly selected to patients with quadriplegia, which could have resulted in a false low incidence of CIN/CIM. Also, approximately $1 / 5$ of patients died within 2 weeks from ICU admission and this probably affected the incidence rate of CIN/CIM. Second, there is a higher proportion of men than women in the COVID-19 cohort, however this predominance of men who acquire a more severe form of the disease is known[36]. Third, the study was done in only one Swedish hospital. Since health-care systems along with treatment strategies vary between hospitals 
and countries, it is not certain that results from high-income countries are directly transferable to low- income and middle-income countries. The finding of elevated NfL and GFAP in patients who later developed CIN/CIM needs to be validated in larger prospective studies, to know how these biomarkers would potentially influence the clinical decisions for these patients. Furthermore, a muscle biopsy was not done in the ICU, and thus the myosin loss as part of CIM could not be assessed.

\section{CONCLUSIONS}

In summary, we found a high proportion of COVID-19 patients with ICUAW who develop CIN. Unlike the previously described cases of COVID-19 patients developing GBS, where the infection most often precede GBS, it is most likely that the lengthy ICU care is an important explanation to the high incidence of CIN. This is important to consider in the differential diagnostic workup as well as the further rehabilitation of these patients. Interestingly we found high early levels of NfL and GFAp suggesting their potentials for predicting CIN/CIM in patients with severe COVID-19. The potential role of NfL, GFAp and tau in axonal nerve pathology in CIN deserves to be further explored.

\section{ABBREVIATIONS}

CIM, critical illness myopathy

CIN, critical illness polyneuropathy

COVID-19, coronavirus disease 2019

GFAp, glial fibrillary acidic protein

ICU, intensive care unit

ICUAW, intensive care unit acquired weakness

NfL, neurofilament light chain

NCS, nerve conduction studies 
SARS-Cov-2, severe acute respiratory syndrome corona virus type 2

\section{Legends to the figures}

Figure 1. Levels of plasma A) neurofilament (NFL), B) glial fibrillary acidic protein (GFAP) and C) Tau in pg/ml (logarithmic scale) comparing patients with CIN/CIM (filled) with the patients without CIN/CIM (open) at the early (median: 4 days, range: 3-9 days) and late timepoint (median: 16 days, range: 11-42 days).

Figure 2. A) Combined motor amplitude (CMA) of all examined motor nerves (ulnar, median, fibular and tibial) and B) combined sensory amplitude (CSA) of all examined sensory nerves (ulnar, radial, sural) in the group of patients without critical illness neuropathy $(\mathrm{CIN})$ or myopathy $(\mathrm{CIM})(\mathrm{N}=3)$ and among patients with $\mathrm{CIN} / \mathrm{CIM}(\mathrm{N}=10)$.

Figure 3. A) Correlation between compound motor amplitude score (CMA) and glial fibrillary acidic protein (GFAp). Spearman $\mathrm{R}=,-0.72 ; \mathrm{p}=0.007$. B) Correlation between compound sensory amplitude score (SCA) and Tau. Spearman $\mathrm{R}=-0.63 ; \mathrm{p}=0.024$.

Supplementary Figure 1. Flow chart of the screening and inclusion of patients in the study. Supplementary Figure 2. Plasma neurofilament (NFL) levels plotted against number of days treated in the ICU divided by CIN/CIM (filled circles) and no CIN/CIM (open circles). 


\section{Ethics approval and consent to participate}

The study was approved by the National Ethical Review Authority (EPM; No. 2020-01623). Informed consent was obtained from the patient, or next of kin if the patient was unable give consent. The Declaration of Helsinki and its subsequent revisions were followed. Electrophysiological data collected from COVID-19 patients and ICU controls were approved by the Ethical Review Board (case no 2015/105 and 2020-03377).

\section{Consent for publication}

Not applicable.

\section{Availability of supporting data}

The datasets analyzed during the current study are available from the corresponding author on reasonable request.

\section{Competing interests}

$\mathrm{KB}$ has served as a consultant, at advisory boards, or at data monitoring committees for Abcam, Axon, Biogen, Julius Clinical, Lilly, MagQu, Novartis, Roche Diagnostics, and Siemens Healthineers, and is a co-founder of Brain Biomarker Solutions in Gothenburg AB (BBS), which is a part of the GU Ventures Incubator Program (outside submitted work). HZ has served at scientific advisory boards for Denali, Roche Diagnostics, Wave, Samumed, Siemens Healthineers, Pinteon Therapeutics and $\operatorname{Cog} R x$, has given lectures in symposia sponsored by Fujirebio, Alzecure and Biogen, and is a co-founder of Brain Biomarker Solutions in Gothenburg AB (BBS), which is a part of the GU Ventures Incubator Program (all outside submitted work). The other authors report no disclosures. 


\section{Funding}

The study was funded by the SciLifeLab/KAW national COVID-19 research program project grant to MH (KAW 2020.0182), and the Swedish Research Council to RF (2014-02569 and 2014-07606). ER is a Wallenberg Clinical Fellow supported by the SciLifeLab/KAW national COVID-19 research program project grant and The Swedish Society of Medicine. KB is supported by the Swedish Research Council (\#2017-00915), the Alzheimer Drug Discovery Foundation (ADDF), USA (\#RDAPB-201809-2016615), the Swedish Alzheimer Foundation (\#AF-742881), Hjärnfonden, Sweden (\#FO2017-0243), the Swedish state under the agreement between the Swedish government and the County Councils, the ALFagreement (\#ALFGBG-715986), and European Union Joint Program for Neurodegenerative Disorders (JPND2019-466-236). HZ is a Wallenberg Scholar supported by grants from the Swedish Research Council (\#2018-02532), the European Research Council (\#681712), Swedish State Support for Clinical Research (\#ALFGBG-720931), the Alzheimer Drug Discovery Foundation (ADDF), USA (\#201809-2016862), and the UK Dementia Research Institute at UCL. ARP is supported by the Swedish Research Council (\#2014-02048 and 2014-07603), Göran Gustafsson Foundation for Medical Research and Hjärnfonden, Sweden (\#FO FO2020-0153).

\section{Authors' contributions}

RF: study design data collection, data analysis, data interpretation, figures, literature search, writing; ER: study design data collection, data analysis, data interpretation, literature search, writing; EK: data collection, data interpretation, writing; JV: data collection, writing; DF: data collection, data interpretation, writing; $\mathrm{MH}$ : data collection, data interpretation, writing; ML: data collection, writing; NA: data collection, data analysis, writing; KB: data analysis, data interpretation, writing; HZ: data analysis, data interpretation, writing; ARP: data collection, data analysis, data interpretation, figures, literature search, writing 


\section{Acknowledgements}

The authors thank research nurses Joanna Wessbergh and Elin Söderman, and the biobank assistants Erik Danielsson and Philip Karlsson for their expertise in compiling the study. The authors thank the physicians and technicians at the department of Clinical Neurophysiology, for excellent electrophysiological work up in the ICU. 


\section{TABLES}

\begin{tabular}{lccccc} 
& $n$ & Covid-19 ICU non-CIN/CIM & $n$ & Covid-19 ICU CIN/CIM & $P$ \\
\hline Gender, male $\mathrm{n}(\%)$ & 100 & $74(74)$ & 11 & $11(100)$ & 0.01 \\
Age, $y r s$ & 100 & $61(51-73)$ & 11 & $64(55-70)$ & 0.97 \\
Body mass index (kg/m2) & 87 & $28(25-32)$ & 10 & $35(30-38)$ & 0.006 \\
ACEi/ARB treatment (\%) & 99 & $36(36)$ & 11 & $5(46)$ & 0.56 \\
& & & & & \\
Comorbidities, n (\%) & & & & $2(18)$ & 0.52 \\
Pulmonary disease & 100 & $27(27)$ & 11 & $7(64)$ & 0.42 \\
Hypertension & 100 & $51(51)$ & 11 & $2(18)$ & 0.07 \\
Heart failure & 100 & $3(3)$ & 11 & $3(27)$ & 0.37 \\
Peripheral vessel disease & 100 & $16(16)$ & 11 & $4(36)$ & 0.43 \\
Diabetes mellitus & 100 & $25(25)$ & 11 & $0(0)$ & 0.25 \\
Neurological disease & 100 & $6(6)$ & 11 & $0(0)$ & 0.19 \\
Malignancy & 100 & $8(8)$ & 11 & & \\
\hline
\end{tabular}

Table 1: Demographic characteristics and comorbidities of 112 patients admitted to intensive care due to severe Covid-19 divided by the development of CIN/CIM or not. ACEi - Angiotensin Converting Enzyme inhibitor; ARB - Angiotensin Receptor Blocker. Values are represented as median (IQR) or n (\%). The p-value originates from the Mann-Whitney $\mathrm{U}$ test for continuous parameters and the chi-square test for categorical parameters. 


\begin{tabular}{lrcccc} 
& \multicolumn{2}{l}{$\begin{array}{l}\text { Covid-19 ICU non- } \\
\text { CIN/CIM }\end{array}$} & $n$ & $\begin{array}{l}\text { Covid-19 ICU } \\
\text { CIN/CIM }\end{array}$ \\
\hline On admission & & & & & \\
SAPS & 97 & $53(47-57)$ & 11 & $50(46-55)$ & 0.40 \\
Respiratory rate (breaths per minute) & 97 & $28(23-36)$ & 11 & $31(26-36)$ & 0.51 \\
Mean arterial blood pressure (mmHg) & 97 & $90(80-98)$ & 11 & $86(79-96)$ & 0.53 \\
Heart rate (beats per minute) & 99 & $89(78-100)$ & 11 & $89(79-101)$ & 0.87 \\
Body temperature (degrees Celsius) & 99 & $38.0(37.4-38.7)$ & 11 & $38.0(37.9-38.6)$ & 0.60 \\
Days between symptoms onset and & 95 & $10(9-13)$ & 11 & $8(8-10.5)$ & 0.11 \\
admission & & & & & \\
& & & & & \\
In the ICU & & & & & \\
Length of stay & 100 & $7(4-12)$ & 11 & $27(18-41)$ & $<0.001$ \\
Thromboembolic events & 100 & $10(10)$ & 11 & $5(46)$ & 0.05 \\
Invasive ventilation (\%) & 100 & $52(52)$ & 11 & $11(100)$ & 0.001 \\
$\quad$ Ventilator free days & 52 & $20(14-24)$ & 11 & $3(0-12)$ & $<0.001$ \\
Vasoactive medication (\%) & 100 & $40(40)$ & 11 & $11(100)$ & 0.002 \\
$\quad$ Vasoactive medication free days & 40 & $20(0-24)$ & 11 & $13(0-17)$ & 0.12 \\
Renal replacement therapy (\%) & 100 & $8(8)$ & 11 & $7(64)$ & 0.003 \\
$\quad$ Renal replacement therapy free days & 8 & $18(0-22)$ & 7 & $9(0-10)$ & 0.19 \\
Continuous propofol anaesthesia (\%) & 100 & $52(52)$ & 11 & $11(100)$ & $<0.001$ \\
$\quad$ Propofol anaesthesia free days & 52 & $21(17-23)$ & 11 & $13(0-15)$ & $<0.001$ \\
Iv Opioid anaelgesia (\%) & 100 & $74(74)$ & 11 & $11(100)$ & 0.01 \\
$\quad$ Iv Opioid anaelgesia free days & 74 & $19(14-24)$ & 11 & $6(0-10)$ & $<0.001$ \\
Continuous iv neurmuscular blockade (\%) & 100 & $45(45)$ & 11 & $11(100)$ & $<0.001$ \\
$\quad$ Iv neurmuscular blockade free days & 45 & $25(21-27)$ & 11 & $22(0-23)$ & $<0.001$ \\
& & & & & \\
ICU-mortality (\%) & 100 & $20(20)$ & 11 & $3(27)$ & 0.58 \\
30-day mortality (\%) & 100 & $25(25)$ & 11 & $2(18)$ & 0.60 \\
\hline
\end{tabular}

Table 2: Patient characteristics and ICU-treatment of 112 patients admitted to intensive care due to severe Covid-19 divided by the development of CIN/CIM or not. Intravenous anesthesia includes propofol and/or midazolam. Opioid analgesia includes morphine, oxycodone, remifentanil and/or fentanyl. Neuromuscular blockade includes rocuronium or atracurium. Values are represented as median (IQR) or n (\%). The p-value originates from the Mann-Whitney U test for continuous parameters and the chi-square test for categorical parameters. Values are represented as median (IQR) or $\mathrm{n}(\%)$. The p-value is calculated for continuous parameters with the Mann-Whitney U test, and for categorical parameters the chi-square test; $\mathrm{p}<$ 0.05 is considered significant. 


\section{Cohort}

\begin{tabular}{|cccc|}
\hline & $\begin{array}{c}\text { COVID-19 } \\
\mathbf{n = 1 4}\end{array}$ & $\begin{array}{c}\text { ICU-ctrl } \\
\mathbf{n = 1 0}\end{array}$ & P \\
\hline Male N (\%) & $13(93 \%)$ & $3(30 \%)$ & $<0.001$ \\
\hline Age (meantSD) & $60.5 \pm 11$ & $66 \pm 13$ & 0.43 \\
CIM & $4(29 \%)$ & $7(70 \%)$ & 0.05 \\
CIN & $7(50 \%)$ & $0(0 \%)$ & 0.008 \\
PNP & 0 & 2 & n.a. \\
\hline Normal findings & 2 & 1 & n.a. \\
\hline Abnormal findings & 1 & 0 & n.a. \\
\hline EMG fib/psw & $9(64 \%)$ & $7(88 \%)$ & 0.25 \\
\hline
\end{tabular}

Table 3. Comparison of the cohort of COVID-19 patients (N=14) and the non-COVID-19 (ICU-ctrl, $\mathrm{N}=10$ ) with regards to $\mathrm{NCS}$ and electromyography (EMG) due to ICU acquired weakness. CIN= critical illness polyneuropathy, ICD-10-code G63.9 and/or CIM= critical illness myopathy, ICD-code G72.9. PNP= polyneuropathy of other cause, ICD-code G62.9; normal findings ICD-code Z01.9; Abnormal, unspecificed abnormal findings. EMG fib/psw, abnormal spontaneous activity including fibrillations and positive sharp waves. N.a.,not assessed due to low number of cases. 


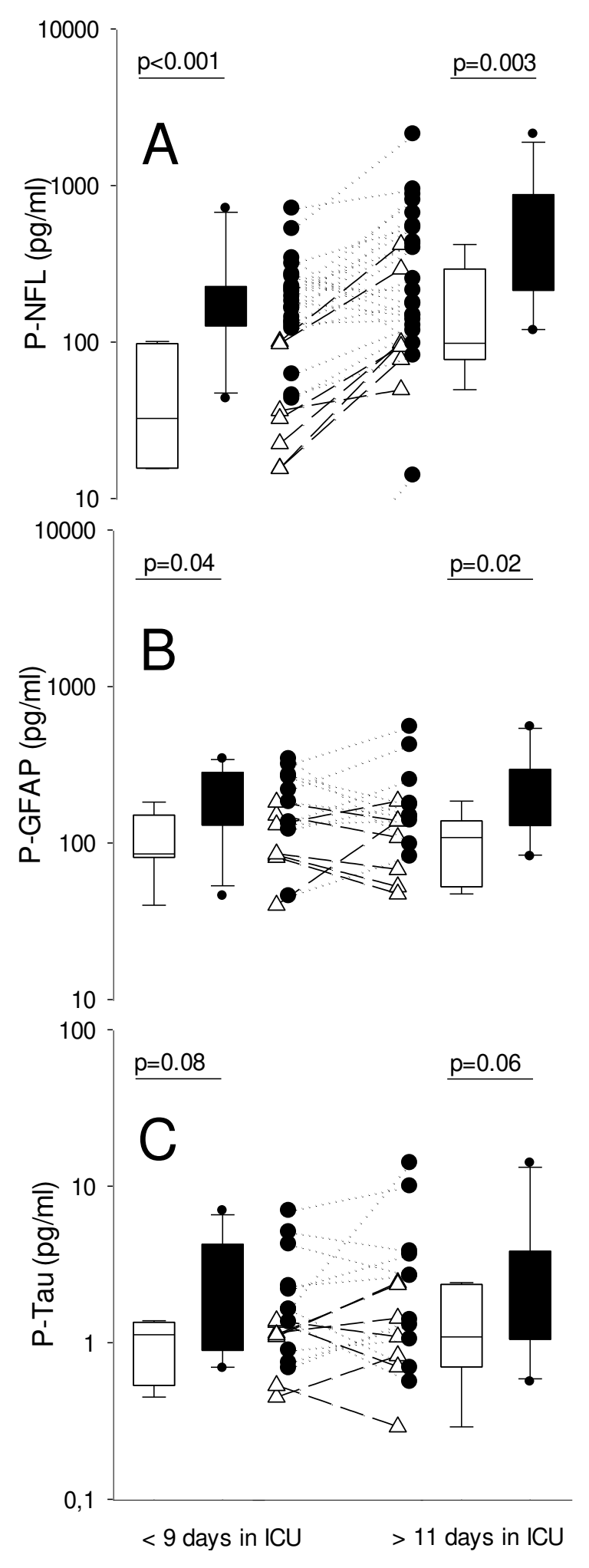


Figure 1. Plasma levels of $\mathbf{A}$ ) neurofilament (NFL), B) glial fibrillary acidic protein (GFAP) and C) Tau in pg/ml (logarithmic scale) comparing patients with CIN/CIM (filled) with the patients without CIN/CIM (open) at the early (median: 4 days, range: 3-9 days) and late timepoint (median: 16 days, range: 11-42 days).
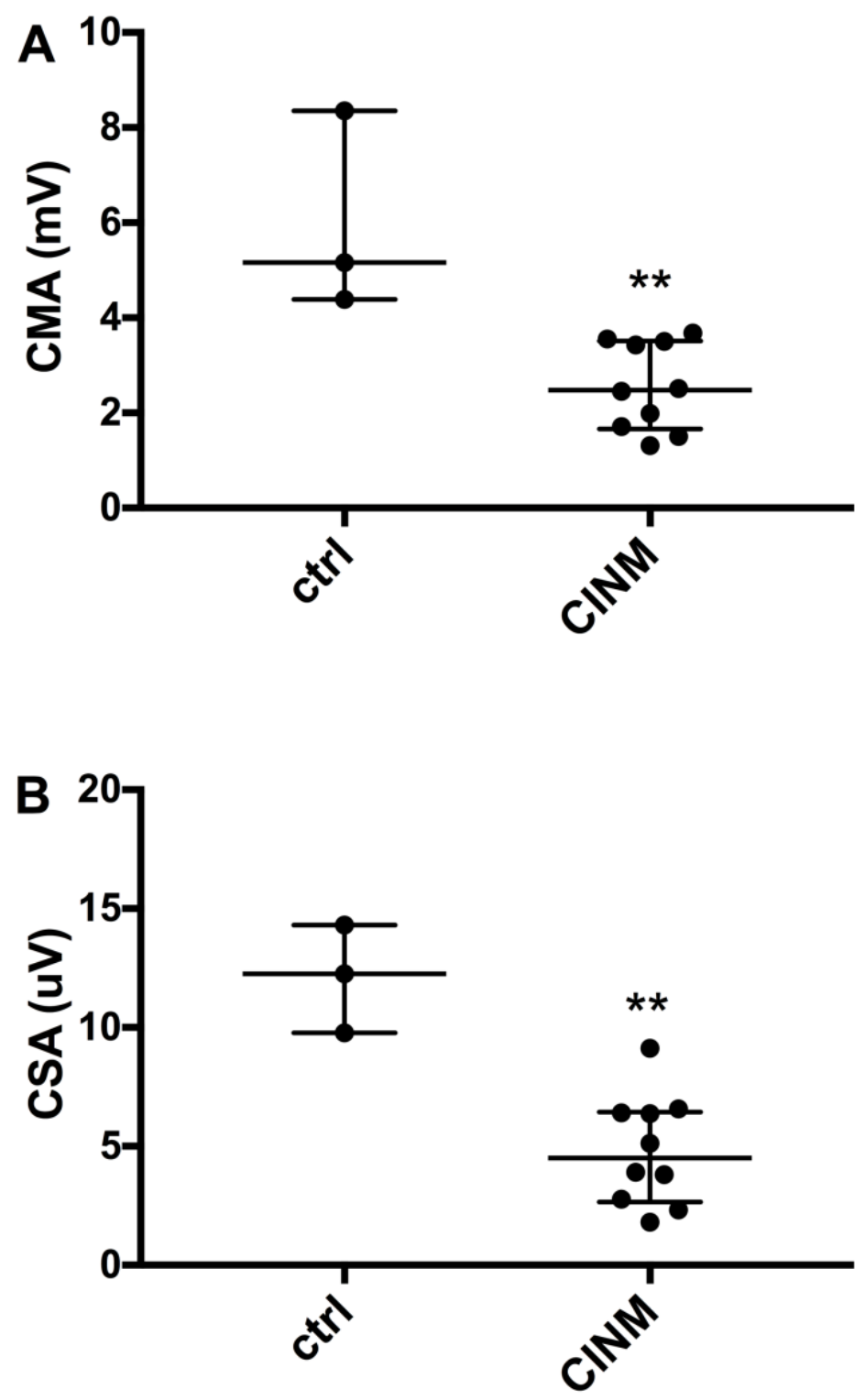

Figure 2. A) Combined motor amplitude (CMA) of all examined motor nerves (ulnar, median, fibular and tibial) and B) combined sensory amplitude (CSA) of all examined sensory nerves (ulnar, radial, sural) in the group of patients without critical illness neuropathy or myopathy (ctrl;;N=3) and among patients with critical illness neuropathy or myopathy (CINM). ${ }^{* *} \mathrm{p}<0.01$. 

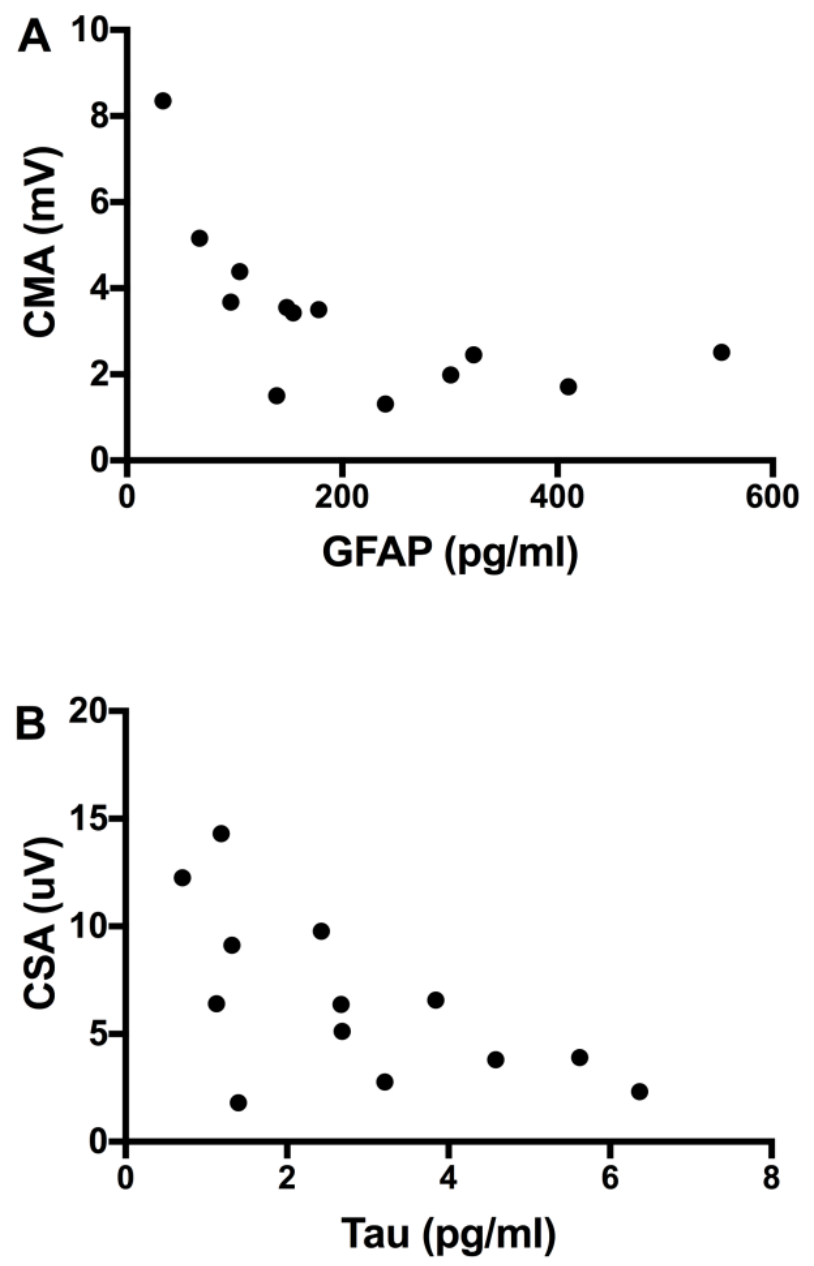

Figure 3. A) Correlation between compound motor amplitude score (CMA) and plasma glial fibrillary acidic protein (GFAP). Spearman $R=,-0.72 ; p=0.007$. B) Correlation between compound sensory amplitude score (SCA) and plasma levels of Tau. Spearman $R=-0.63 ; p=0.024$. 


\section{REFERENCES}

1. Ellul MA, Benjamin L, Singh B, Lant S, Michael BD, Easton A, Kneen R, Defres S, Sejvar J, Solomon T: Neurological associations of COVID-19. Lancet Neurol 2020.

2. Mao L, Jin H, Wang M, Hu Y, Chen S, He Q, Chang J, Hong C, Zhou Y, Wang D et al: Neurologic Manifestations of Hospitalized Patients With Coronavirus Disease 2019 in Wuhan, China. JAMA Neurol 2020.

3. Tsai LK, Hsieh ST, Chao CC, Chen YC, Lin YH, Chang SC, Chang YC:

Neuromuscular disorders in severe acute respiratory syndrome. Arch Neurol 2004, 61(11):1669-1673.

4. Camdessanche JP, Morel J, Pozzetto B, Paul S, Tholance Y, Botelho-Nevers E: COVID-19 may induce Guillain-Barre syndrome. Rev Neurol (Paris) 2020, 176(6):516518.

5. Toscano G, Palmerini F, Ravaglia S, Ruiz L, Invernizzi P, Cuzzoni MG, Franciotta D, Baldanti F, Daturi R, Postorino P et al: Guillain-Barre Syndrome Associated with SARSCoV-2. N Engl J Med 2020, 382(26):2574-2576.

6. Zhao H, Shen D, Zhou H, Liu J, Chen S: Guillain-Barre syndrome associated with SARS-CoV-2 infection: causality or coincidence? Lancet Neurol 2020, 19(5):383-384.

7. Latronico N, Bolton CF: Critical illness polyneuropathy and myopathy: a major cause of muscle weakness and paralysis. Lancet Neurol 2011, 10(10):931-941.

8. Hanidziar D, Bittner E: Sedation of mechanically ventilated COVID-19 patients: challenges and special considerations. Anesth Analg 2020.

9. Herridge MS, Tansey CM, Matte A, Tomlinson G, Diaz-Granados N, Cooper A, Guest CB, Mazer CD, Mehta S, Stewart TE et al: Functional disability 5 years after acute respiratory distress syndrome. $N$ Engl J Med 2011, 364(14):1293-1304. 
10. Iwashyna TJ: Survivorship will be the defining challenge of critical care in the 21st century. Ann Intern Med 2010, 153(3):204-205.

11. Leijten FS, Harinck-de Weerd JE, Poortvliet DC, de Weerd AW: The role of polyneuropathy in motor convalescence after prolonged mechanical ventilation. JAMA 1995, 274(15):1221-1225.

12. Guarneri B, Bertolini G, Latronico N: Long-term outcome in patients with critical illness myopathy or neuropathy: the Italian multicentre CRIMYNE study. $J$ Neurol Neurosurg Psychiatry 2008, 79(7):838-841.

13. Tankisi H, Tankisi A, Harbo T, Markvardsen LK, Andersen H, Pedersen TH: Critical illness myopathy as a consequence of Covid-19 infection. Clin Neurophysiol 2020, 131(8):1931-1932.

14. Wieske L, Witteveen E, Petzold A, Verhamme C, Schultz MJ, van Schaik IN, Horn J: Neurofilaments as a plasma biomarker for ICU-acquired weakness: an observational pilot study. Crit Care 2014, 18(1):R18.

15. Corman VM, Landt O, Kaiser M, Molenkamp R, Meijer A, Chu DK, Bleicker T, Brunink S, Schneider J, Schmidt ML et al: Detection of 2019 novel coronavirus (2019nCoV) by real-time RT-PCR. Euro Surveill 2020, 25(3).

16. Bolton CF: Neuromuscular manifestations of critical illness. Muscle Nerve 2005, 32(2):140-163.

17. Lacomis D, Zochodne DW, Bird SJ: Critical illness myopathy. Muscle Nerve 2000, 23(12):1785-1788.

18. Kanberg N, Ashton NJ, Andersson LM, Yilmaz A, Lindh M, Nilsson S, Price RW, Blennow K, Zetterberg H, Gisslen M: Neurochemical evidence of astrocytic and neuronal injury commonly found in COVID-19. Neurology 2020. 
19. Z'Graggen WJ, Tankisi H: Critical Illness Myopathy. J Clin Neurophysiol 2020, 37(3):200-204.

20. Lonnqvist PA, Bell M, Karlsson T, Wiklund L, Hoglund AS, Larsson L: Does prolonged propofol sedation of mechanically ventilated COVID-19 patients contribute to critical illness myopathy? Br J Anaesth 2020.

21. Tennila A, Salmi T, Pettila V, Roine RO, Varpula T, Takkunen O: Early signs of critical illness polyneuropathy in ICU patients with systemic inflammatory response syndrome or sepsis. Intensive Care Med 2000, 26(9):1360-1363.

22. Steinberg KP, Hudson LD, Goodman RB, Hough CL, Lanken PN, Hyzy R, Thompson BT, Ancukiewicz M, National Heart L, Blood Institute Acute Respiratory Distress Syndrome Clinical Trials N: Efficacy and safety of corticosteroids for persistent acute respiratory distress syndrome. $N$ Engl $J$ Med 2006, 354(16):1671-1684.

23. Bercker S, Weber-Carstens S, Deja M, Grimm C, Wolf S, Behse F, Busch T, Falke KJ, Kaisers U: Critical illness polyneuropathy and myopathy in patients with acute respiratory distress syndrome. Crit Care Med 2005, 33(4):711-715.

24. De Jonghe B, Sharshar T, Lefaucheur JP, Authier FJ, Durand-Zaleski I, Boussarsar M, Cerf C, Renaud E, Mesrati F, Carlet $\mathrm{J}$ et al: Paresis acquired in the intensive care unit: a prospective multicenter study. JAMA 2002, 288(22):2859-2867.

25. Yang $\mathrm{T}$, Li Z, Jiang L, Wang Y, Xi X: Risk factors for intensive care unit-acquired weakness: A systematic review and meta-analysis. Acta Neurol Scand 2018, 138(2):104114.

26. Latronico N, Peli E, Botteri M: Critical illness myopathy and neuropathy. Curr Opin Crit Care 2005, 11(2):126-132.

27. Zimnowodzki S, Butrum M, Kimura J, Stalberg E, Mahajan S, Gao L: Emergence of F-waves after repetitive nerve stimulation. Clin Neurophysiol Pract 2020, 5:100-103. 
28. Novak KR, Nardelli P, Cope TC, Filatov G, Glass JD, Khan J, Rich MM:

Inactivation of sodium channels underlies reversible neuropathy during critical illness in rats. $J$ Clin Invest 2009, 119(5):1150-1158.

29. Gaetani L, Hoglund K, Parnetti L, Pujol-Calderon F, Becker B, Eusebi P, Sarchielli P, Calabresi P, Di Filippo M, Zetterberg H et al: A new enzyme-linked immunosorbent assay for neurofilament light in cerebrospinal fluid: analytical validation and clinical evaluation. Alzheimers Res Ther 2018, 10(1):8.

30. Chelban V, Wilson MP, Warman Chardon J, Vandrovcova J, Zanetti MN, ZambaPapanicolaou E, Efthymiou S, Pope S, Conte MR, Abis G et al: PDXK mutations cause polyneuropathy responsive to pyridoxal 5'-phosphate supplementation. Ann Neurol 2019, 86(2):225-240.

31. Kapoor M, Foiani M, Heslegrave A, Zetterberg H, Lunn MP, Malaspina A, Gillmore JD, Rossor AM, Reilly MM: Plasma neurofilament light chain concentration is increased and correlates with the severity of neuropathy in hereditary transthyretin amyloidosis. J Peripher Nerv Syst 2019, 24(4):314-319.

32. Sandelius A, Zetterberg H, Blennow K, Adiutori R, Malaspina A, Laura M, Reilly MM, Rossor AM: Plasma neurofilament light chain concentration in the inherited peripheral neuropathies. Neurology 2018, 90(6):e518-e524.

33. Olsson B, Lautner R, Andreasson U, Ohrfelt A, Portelius E, Bjerke M, Holtta M, Rosen C, Olsson C, Strobel G et al: CSF and blood biomarkers for the diagnosis of Alzheimer's disease: a systematic review and meta-analysis. Lancet Neurol 2016, 15(7):673-684.

34. Scholl M, Maass A, Mattsson N, Ashton NJ, Blennow K, Zetterberg H, Jagust W: Biomarkers for tau pathology. Mol Cell Neurosci 2019, 97:18-33. 
35. Caprelli MT, Mothe AJ, Tator CH: Hyperphosphorylated Tau as a Novel Biomarker for Traumatic Axonal Injury in the Spinal Cord. J Neurotrauma 2018, 35(16):1929-1941.

36. Gebhard C, Regitz-Zagrosek V, Neuhauser HK, Morgan R, Klein SL: Impact of sex and gender on COVID-19 outcomes in Europe. Biol Sex Differ 2020, 11(1):29. 
Figures



\section{Figure 1}

Plasma levels of A) neurofilament (NFL), B) glial fibrillary acidic protein (GFAP) and C) Tau in $\mathrm{pg} / \mathrm{ml}$ (logarithmic scale) comparing patients with $\mathrm{CIN} / \mathrm{CIM}$ (filled) with the patients without CIN/CIM (open) at the early (median: 4 days, range: 3-9 days) and late timepoint (median: 16 days, range: 11-42 days). 

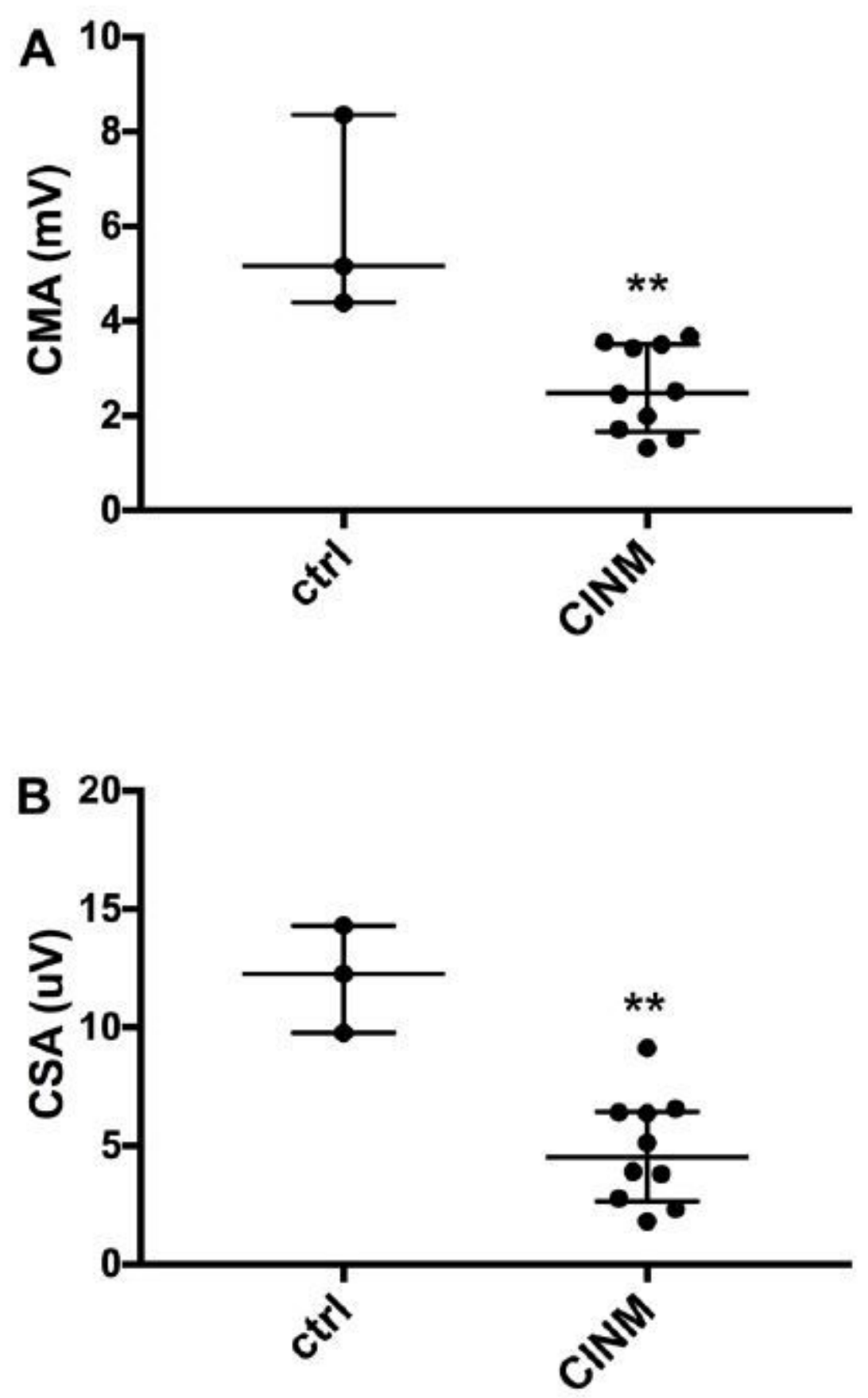

Figure 2

A) Combined motor amplitude (CMA) of all examined motor nerves (ulnar, median, fibular and tibial) and B) combined sensory amplitude (CSA) of all examined sensory nerves (ulnar, radial, sural) in the group of patients without critical illness neuropathy or myopathy (ctrl;; $\mathrm{N}=3$ ) and among patients with critical illness neuropathy or myopathy (CINM). ** $p<0.01$. 

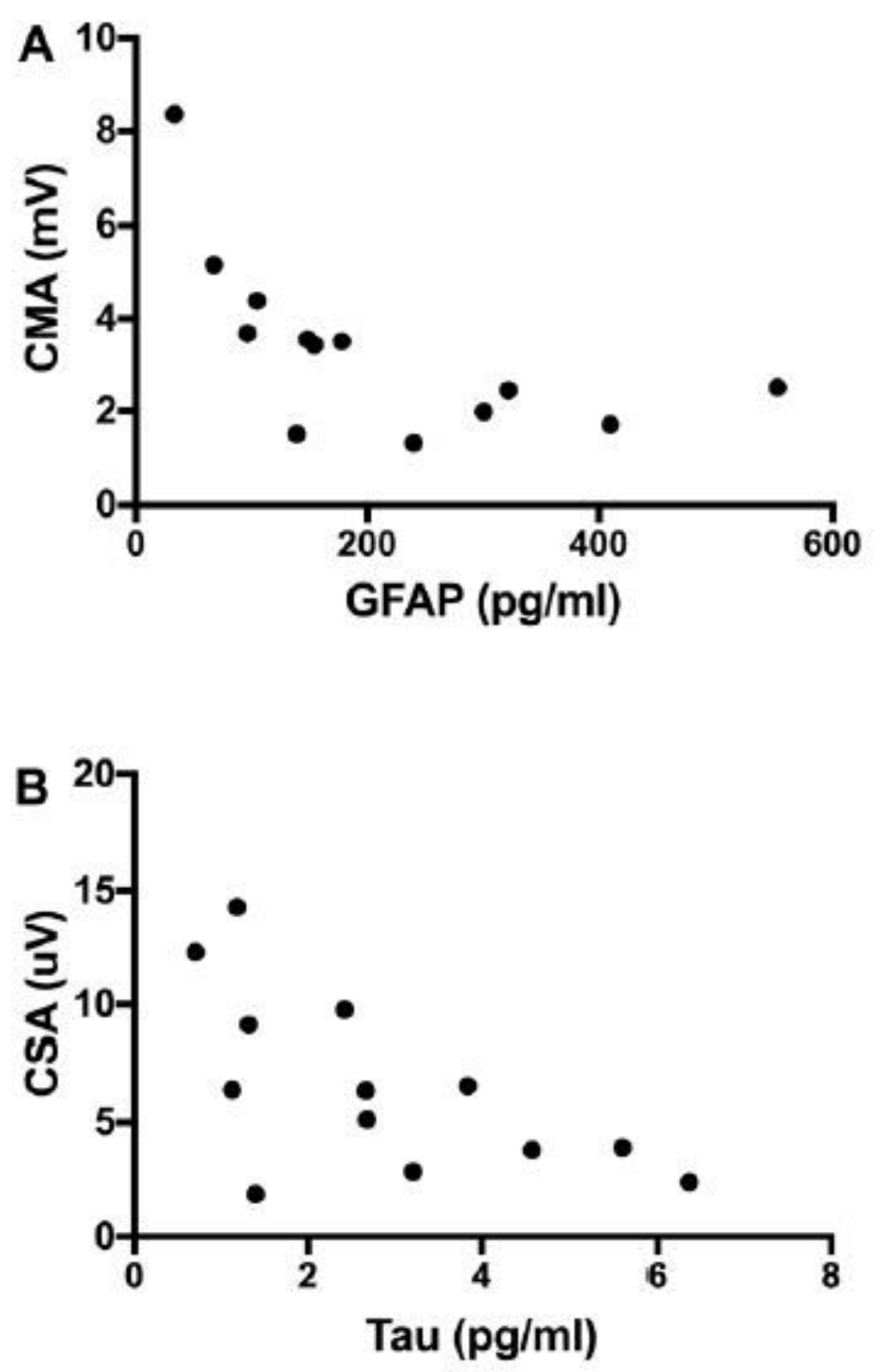

Figure 3

A) Correlation between compound motor amplitude score (CMA) and plasma glial fibrillary acidic protein (GFAP). Spearman $R=,-0.72 ; p=0.007$. B) Correlation between compound sensory amplitude score (SCA) and plasma levels of Tau. Spearman $R=-0.63 ; p=0.024$.

\section{Supplementary Files}

This is a list of supplementary files associated with this preprint. Click to download.

- supplementaryfigure2rev.tif

- supplfig1Flowchart.tif 
- supplementarytables.pdf 